ISSN: 2386-3919 - e-ISSN: 2386-3927

DOI: https://doi.org/10.14201/et2018361179197

\title{
EL APRENDIZAJE DE VALORES A TRAVÉS DE LOS CUENTOS EN EDUCACIÓN INFANTIL
}

\section{Values learning througth tales in chilhood education}

\author{
V. Marín Díaz y C. SÁNChez Cuenca
}

Recibido: 29.10.2017; Aceptado: 10.01.2018; Publicado: 30.06.2018

Ref. Bibl. V. MARÍN DÍAZ y C. SÁNCHEZ CUENCA. El aprendizaje de valores a través de los cuentos en Educación Infantil. Enseñanza \& Teaching, 36, 1-2018, 179-197.

RESUMEN: El aprendizaje de los valores establecidos en la sociedad actual es vital para el crecimiento del individuo de manera amoldada a lo que hoy prima en la comunidad en la que vive. Su instrucción está supeditada a una formación previa, la cual se inicia en la etapa de Educación Infantil. Por otra parte, la enseñanza en este nivel se apoya en la lectura de cuentos tradicionales, los cuales tiene una gran representación de los valores socialmente aceptados hoy, de ahí que la educación en valores cobre una gran relevancia y sea necesario un estudio en este nivel escolar. Mediante un estudio ex post facto, empleando un diseño descriptivo y uno comparativo-causal, se presenta la opinión de los maestros y docentes en preservicio de esta etapa académica de la provincia de Córdoba $(\mathrm{N}=441)$, en torno a si el valor de la cooperación y/o ayuda mutua puede ser aprendido por los niños, a través de los cuentos y del empleo de metodologías tradicionales. Mediante la aplicación de un cuestionario creado ad hoc conformado por 19 cuentos y 5 valores, atendiendo a la clasificación realizada por Marín y Sánchez (2015), y empleando una escala de respuesta tipo Likert, donde 1 equivalía a totalmente en desacuerdo y 5 a totalmente en desacuerdo, se procedió a la recogida de los datos. El principal resultado alcanzado es que los docentes más jóvenes consideran que todos los cuentos trabajados en la enseñanza infantil (19) son válidos para aprender dicho valor. En consecuencia, cabe preguntarse si la falta de experiencia profesional puede ser un elemento que determine el empleo de metodologías tradicionales para el aprendizaje de este valor en particular y de todos en general. 
Palabras clave: valores; aprendizaje; metodología tradicional; cooperación; ayuda mutua.

SUMMARY: The learning of values established in today's society is vital for the growth of the individual in a way that today is a premium in the community in which their live. Their learning is dependent at previous train, which it begins in childhood education level. In the other hand, teaching at this level is supported on the reading of traditional tales, which has a great representation of the values socially accepted today, hence that education in values had a great relevant and needs a study in this School level. By a ex post facto study, used a descriptive an comparative-casual design, be presented the opinion of teachers and pre-service teachers on this academic level in the Cordoba province $(\mathrm{N}=411)$, about if the value of cooperation and/or mutual help can be learned by the children through the tales and used of traditional methodologies. Through the application a questionnaire create ad hoc conformed with 19 tales and 5 values, according to classification made by Marín and Sánchez (2015) and used a scale to scale type Likert to answered, where 1 was totally disagree and 5 totally in disagreement, it preceded the collection of data. The principal results are the younger teachers consider that all the tales offered (19) are valid to learn this value. In consequence we can question, if lack of professional experience can be an element that determines the use of traditional methodologies for the learning of this particular value and all in general.

Key words: values; learning; traditional methodology; cooperation; mutual help.

\section{INTRODUCCIÓN}

Leer y escribir son dos destrezas básicas para el desarrollo del individuo no solo como persona, sino también como sujeto activo de la sociedad en la que vive. Como afirma Gutiérrez (2016), la lectura es un acto cargado de creatividad, la cual en los estadios iniciales del proceso formativo va a ser el eje sobre el que pivote la socialización de los infantes. A la vez, durante el transcurso del aprendizaje de la acción lectora, se van adquiriendo y transmitiendo los valores que la sociedad tiene establecidos como positivos y negativos (antivalores o contravalores); del mismo modo se perfilan los estereotipos que van a marcar la forma de ver, sentir y entender la vida. Algunos autores como Duque y Ovalle (2012) apuntan que la lectura es en sí misma un valor, y ahí es donde, a nuestro juicio, radica la importancia de su empleo desde la primera infancia.

La actual sociedad no puede ser vivida de espaldas a ella, se ha de tratar de formar a los futuros líderes del mundo en una educación ética y moral, que les haga valedores de la fuerza necesaria para saber afrontar las situaciones en las que su sistema de creencias y valores se ponga en jaque. Es por ello que la lectura debe ser entendida como herramienta mediadora del aprendizaje de los valores que hoy son aceptados por la comunidad. 
Como afirma Sáez (2015), los valores existen desde que el hombre tiene conciencia, lo cual unido al lenguaje permite a los individuos emitir juicios y opiniones; es por ello que se considera que durante la etapa de Educación Infantil sean cruciales para su adquisición y/o aprendizaje.

La educación de hoy, en general, ha de saber dar respuesta a una sociedad heterogénea, lo cual conlleva la creación de un esquema de valores (Parra, 2003), por lo que hablar de educación en valores en la etapa inicial de la formación implica hacerlo del empleo de los cuentos, dado que estos son el medio por el cual los contenidos curriculares son introducidos en la capacitación de los infantes.

Si entendemos, de un lado, que la educación en valores «es el proceso mediante el cual se guía y se forma a los alumnos para que sean capaces de utilizar su experiencia axiológica de manera consciente para construir su proyecto personal de vida" (Merma, Perió y Gavila, 2013: 156) y, de otro, que el cuento según Padial y Sáenz-López (2013: 31) pueda ser entendido como recurso pedagógico, que puede servir para "divertir y entretener a la vez que trasmite conocimientos ricos y complejos..., conecta con las características cognitivas-afectivas de niños y niñas..., facilita la superación del egocentrismo..., prepara para la vida ofreciendo modelos de comportamiento, sentimientos y valores", entenderemos que en las etapas iniciales de la formación los cuentos se conviertan en el recurso sobre el que gire la educación en valores.

Compartimos con Lawrence y Pauge (2016) que las historias están construidas en base a nuestras propias experiencias y no son la acumulación lineal de acontecimientos, es por ello que entendemos que el binomio educación en valores-cuentos es la combinación sobre la que la enseñanza inicial debe apoyarse, de cara a la creación de una generación acorde con el momento social actual.

A través de esta investigación, presentamos los resultados alcanzados en torno a la posibilidad que pueden tener los cuentos empleados en la primera infancia para transmitir valores, a juicio de los docentes que trabajan en dicha etapa educativa, así como de los que en pocos meses se iniciarán en ella y que están en último año de su formación inicial, pero que ya han adquirido las competencias y saberes didáctico-pedagógicos para ejercer la profesión, maestros en preservicio. Para ello se ha seleccionado en concreto el valor de la cooperación y/o la ayuda. Se ha centrado este trabajo en este especialmente, por la situación mundial que hoy se está viviendo en torno a los movimientos migratorios generados por las situaciones de precariedad económica o falta de seguridad de los países.

\section{MÉTODO}

El interrogante de partida de esta investigación es saber ¿cuál es la opinión de los docentes, en ejercicio y en formación inicial final, de la etapa de Educación Infantil sobre el valor de los cuentos a través de metodologías tradicional, en la transmisión de valores de los menores? 
El objetivo general emanado de este interrogante ha sido determinar la opinión de los docentes, tanto en ejercicio como en formación inicial, sobre la transmisión de valores en diferentes narrativas infantiles. De este, nace como objetivo específico: conocer la opinión de los profesionales de la etapa de infantil, tanto de los que imparten como los que se encuentran en formación inicial, y de apoyo sobre la transmisión del valor de cooperación y ayuda mediante distintos cuentos a través de metodologías tradicionales (lectura del mismo por parte del docente, visionado y coloreado de láminas, escenificación del cuento, etc.).

El método empleado ha sido el ex post facto, utilizando el diseño descriptivo y comparativo-causal (Mateo, 2012).

\subsection{Selección de la muestra}

La población objeto de estudio de esta investigación contempla dos subgrupos bien definidos, por un lado, el alumnado o maestros en preservicio de cuarto curso del Grado de Educación Infantil de la Facultad de Ciencias de la Educación de la Universidad de Córdoba; y, por otro, los maestros en ejercicio de la etapa de Educación Infantil de la población o núcleo urbano de la ciudad de Córdoba.

El número de población asciende a 441 sujetos como sumatorio de la combinación del subconjunto poblacional de los docentes $\left(\mathrm{N}^{1}=261\right)$ y, el de los maestros en preservicio [estudiantes] $\left(\mathrm{N}^{2}=180\right)$, con un nivel de confianza del $95 \%$ y una varianza relativa de $\mathrm{z}=1.96$ y una heterogeneidad de la población del 50\%, concurriendo estos datos como los mínimos estandarizados y, al aplicar la fórmula expresada por Morales (2012) para conocer el margen de error, el resultado que provoca la misma es del 3.3\%; corroborando el mismo un error muestral aceptable de la muestra inicial recogida $(\mathrm{N}=298$ sujetos $)$.

El método de selección de la muestra ha sido de tipo estratificado (Sabariego, 2012), dividiendo en primer lugar la población en dos estratos (docentes y maestros en preservicio [estudiantes]); determinando seguidamente el tamaño de la muestra; dentro de cada estrato se ha ejecutado un muestreo aleatorio simple; y, finalmente, el número de sujetos de cada estrato se ha decidido por afijación proporcional, atendiendo a la proporción de individuos de cada estrato.

1. El número exacto de la población ha sido facilitado por la Consejería Provincial de Educación de Córdoba para el curso académico (2015/2016) atendiendo a los datos estadísticos que obran en este organismo para maestros del $2{ }^{\circ}$ ciclo de la etapa de infantil que esté ejerciendo en la ciudad cordobesa.

2. El dato relativo a la población de estudiantes de cuarto curso del Grado de Educación Infantil ha sido suministrado por la Secretaría de la Facultad de Ciencias de la Educación de la Universidad de Córdoba. 
V. MARÍN DÍAZ Y C. SÁNCHEZ CUENCA

EL APRENDIZAJE DE VALORES A TRAVÉS DE LOS CUENTOS EN EDUCACIÓN INFANTIL

TABLA 1

Porcentaje de la muestra atendiendo a la población

\begin{tabular}{|l|c|c|c|}
\hline \multicolumn{1}{|c|}{ ESTRATOS } & $\begin{array}{c}\text { N. }^{\text {o }} \text { DE SUJETOS ATENDIENDO } \\
\text { A LAS FUENTES CONSULTADAS }\end{array}$ & $\begin{array}{c}\text { N. }^{\circ} \text { DE SUJETOS } \\
\text { ENCUESTADOS }\end{array}$ & $\%$ \\
\hline Docentes & 261 & 173 & $66.3 \%$ \\
\hline Maestros en preservicio & 180 & 125 & $69.4 \%$ \\
\hline Total & 441 & 298 & $67.6 \%$ \\
\hline
\end{tabular}

Finalmente, la muestra está constituida por 217 sujetos, de los cuales el $4.7 \%$ son hombres y el $95.3 \%$ mujeres. Los encuestados, respecto de las edades, el $40.1 \%$ son menores de 25 años; el 4.6\% en el intervalo de edad de 26 a 30 años; 8.3 de cada 100 participantes tienen entre 31 y 35 años; el 9.2\% de 36 a 40 años; los de 41 a 45 años son el 13.8\%; y el 24.0\% más de 46 años. Atendiendo a su situación profesional, encontramos que el $41.9 \%$ eran estudiantes en formación inicial de 4 . $^{\circ}$ curso y el $58.1 \%$ eran profesores en ejercicio.

\subsection{Diseño del instrumento}

La determinación de los cuentos empleados ha girado en torno a la clasificación realizada por Marín-Díaz y Sánchez-Cuenca (2015: 1098), establecida en función de la edad de los estudiantes de dicha etapa formativa. Los 19 cuentos seleccionados (El patito feo; Caperucita Roja; El flautista de Hamelin; Hansel y Gretel; La bella durmiente; El gato con botas; Los tres cerditos; Peter Pan; La Cenicienta; Los siete cabritillos; Los tres osos; Pulgarcito; Pinocho; Blancanieves; Bambi; La lechera; El mago de Oz; La bella y la bestia; El libro de la selva) se han agrupado en torno a los 5 valores (cooperación y/o ayuda, justicia, respeto mutuo, responsabilidad y tolerancia de carácter general) aportados por la investigación de las autoras. El instrumento quedó finalmente conformado por una escala de valoración de cada cuento para cada dimensión o valores (19 cuentos x 5 valores), con una escala de respuesta tipo Likert de 5 opciones, donde 1 significaba no se transmite totalmente y 5 se transmite totalmente.

Para determinar la fiabilidad del instrumento se realizó la prueba Alfa de Cronbach, para todos los valores, arrojando una puntuación 0.981. En lo que se refiere a la dimensión del valor cooperación y/o ayuda mutua, la puntuación fue 0.918. Por lo que, atendiendo a Mateo (2012), podemos determinar que el instrumento tiene una fiabilidad muy alta. 


\section{RESUlTAdos}

A continuación, se muestran los resultados obtenidos en la opinión de los participantes en relación a la transmisión del valor de cooperación y/o ayuda de los 19 cuentos analizados mediante metodologías tradicionales.

En general, los participantes tienden a mostrarse indiferentes -ni en desacuerdo ni de acuerdo-, con la idea de que los 19 cuentos tradicionales transmiten el valor de la cooperación y/o ayuda mediante una metodología basada en recursos tradicionales; sin embargo, esta opinión tiene valores más altos en las narrativas infantiles de Los tres cerditos $(\overline{\mathrm{x}}=3.08)$; El libro de la selva $(\overline{\mathrm{x}}=3.02)$; Peter Pan $(\overline{\mathrm{x}}=3.01)$; Hansel y Gretel y Blancanieves (ambos $\overline{\mathrm{x}}=3.00)$; y más bajos, en las obras literarias infantiles como La lechera $(\overline{\mathrm{x}}=2.68)$, Caperucita Roja $(\overline{\mathrm{x}}=2.74)$, y El patito feo $(\overline{\mathrm{x}}=2.78)$.

\section{TABLA 2}

Transmisión del valor cooperación y/o ayuda en los cuentos mediante metodologías tradicionales

\begin{tabular}{|l|c|c|c|}
\hline \multicolumn{1}{|c|}{ CuENTO } & N & MEDIA & S \\
\hline El patito feo & 213 & 2.78 & 1.290 \\
\hline Caperucita Roja & 213 & 2.74 & 1.299 \\
\hline El flautista de Hamelín & 213 & 2.81 & 1.277 \\
\hline Hansel y Gretel & 213 & 3.00 & 1.409 \\
\hline La bella durmiente & 213 & 2.79 & 1.323 \\
\hline El gato con botas & 213 & 2.81 & 1.352 \\
\hline Los tres cerditos & 213 & 3.08 & 1.504 \\
\hline Peter Pan & 213 & 3.01 & 1.447 \\
\hline La Cenicienta & 213 & 2.92 & 1.433 \\
\hline Los siete cabritillos & 213 & 2.85 & 1.353 \\
\hline Los tres osos & 213 & 2.81 & 1.341 \\
\hline Pulgarcito & 213 & 2.88 & 1.370 \\
\hline Pinocho & 213 & 2.83 & 1.393 \\
\hline Blancanieves & 213 & 3.00 & 1.398 \\
\hline Bambi & 213 & 2.88 & 1.359 \\
\hline La lechera & 213 & 2.68 & 1.264 \\
\hline El mago de Oz & 213 & 2.80 & 1.357 \\
\hline La bella y la bestia & 213 & 2.89 & 1.357 \\
\hline El libro de la selva & 213 & 3.02 & 1.418 \\
\hline
\end{tabular}


El análisis de comparaciones entre la opinión de los sujetos participantes en relación a la transmisión del valor cooperación y/o ayuda en los cuentos mediante una metodología con recursos tradicionales y el sexo de los mismos se ha ejecutado a través de la prueba inferencial T de Student $(n . s .=0.05)$, la cual provoca los siguientes resultados significativos:

TABLA 3

T de Student de la opinión de la transmisión de la cooperación y/o ayuda en los cuentos mediante metodología tradicional en función del sexo de los participantes

\begin{tabular}{|c|c|c|c|c|c|}
\hline Cuento & SEXO & $\mathrm{N}$ & MEDIA & s & T Y P \\
\hline \multirow{2}{*}{ El patito feo } & Hombre & 10 & 3.30 & 1.252 & \multirow{2}{*}{$\begin{array}{l}\mathrm{T}=1.304 \mathrm{y} \\
\mathrm{p}=0.194 \mathrm{no} \\
\text { significativa }\end{array}$} \\
\hline & Mujer & 203 & 2.76 & 1.289 & \\
\hline \multirow{2}{*}{ Caperucita Roja } & Hombre & 10 & 2.90 & 1.792 & \multirow{2}{*}{$\begin{array}{l}\mathrm{T}=0.286 \mathrm{y} \\
\mathrm{p}=0.781 \text { no } \\
\text { significativa }\end{array}$} \\
\hline & Mujer & 203 & 2.74 & 1.274 & \\
\hline \multirow{2}{*}{$\begin{array}{l}\text { El flautista de } \\
\text { Hamelín }\end{array}$} & Hombre & 10 & 3.90 & 1.287 & \multirow{2}{*}{$\begin{array}{l}\mathrm{T}=2.821 \mathrm{y} \\
\mathrm{p}=0.005 \text { a favor } \\
\text { de hombre }\end{array}$} \\
\hline & Mujer & 203 & 2.75 & 1.254 & \\
\hline \multirow{2}{*}{ Hansel y Gretel } & Hombre & 10 & 4.00 & 1.333 & \multirow{2}{*}{$\begin{array}{l}\mathrm{T}=2.339 \mathrm{y} \\
\mathrm{p}=0.020 \text { a favor } \\
\text { de hombre }\end{array}$} \\
\hline & Mujer & 203 & 2.94 & 1.396 & \\
\hline \multirow[b]{2}{*}{ La bella durmiente } & Hombre & 10 & 3.50 & 1.354 & \multirow{2}{*}{$\begin{array}{l}\mathrm{T}=1.757 \mathrm{y} \\
\mathrm{p}=0.080 \text { no } \\
\text { significativa }\end{array}$} \\
\hline & Mujer & 203 & 2.75 & 1.314 & \\
\hline \multirow{2}{*}{ El gato con botas } & Hombre & 10 & 3.80 & 1.317 & \multirow{2}{*}{$\begin{array}{l}\mathrm{T}=2.414 \mathrm{y} \\
\mathrm{p}=0.017 \text { a favor } \\
\text { de hombre }\end{array}$} \\
\hline & Mujer & 203 & 2.75 & 1.337 & \\
\hline \multirow[b]{2}{*}{ Los tres cerditos } & Hombre & 10 & 4.10 & 1.287 & \multirow{2}{*}{$\begin{array}{l}\mathrm{T}=2.224 \mathrm{y} \\
\mathrm{p}=0.027 \text { a favor } \\
\text { de hombre }\end{array}$} \\
\hline & Mujer & 203 & 3.03 & 1.498 & \\
\hline \multirow{2}{*}{ Peter Pan } & Hombre & 10 & 3.70 & 1.337 & \multirow{2}{*}{$\begin{array}{l}\mathrm{T}=1.541 \mathrm{y} \\
\mathrm{p}=0.125 \mathrm{no} \\
\text { significativa }\end{array}$} \\
\hline & Mujer & 203 & 2.98 & 1.447 & \\
\hline \multirow{2}{*}{ La Cenicienta } & Hombre & 10 & 3.70 & 1.252 & \multirow{2}{*}{$\begin{array}{l}\mathrm{T}=1.772 \mathrm{y} \\
\mathrm{p}=0.078 \text { no } \\
\text { significativa }\end{array}$} \\
\hline & Mujer & 203 & 2.88 & 1.433 & \\
\hline \multirow{2}{*}{ Los siete cabritillos } & Hombre & 10 & 3.80 & 1.317 & \multirow{2}{*}{$\begin{array}{l}\mathrm{T}=2.296 \mathrm{y} \\
\mathrm{p}=0.023 \text { a favor } \\
\text { de hombre }\end{array}$} \\
\hline & Mujer & 203 & 2.80 & 1.340 & \\
\hline
\end{tabular}




\begin{tabular}{|c|c|c|c|c|c|}
\hline CUENTO & SEXO & $\mathbf{N}$ & MEDIA & $\mathrm{s}$ & T Y P \\
\hline \multirow{2}{*}{ Los tres osos } & Hombre & 10 & 3.70 & 1.337 & \multirow{2}{*}{$\begin{array}{l}\mathrm{T}=2.180 \mathrm{y} \\
\mathrm{p}=0.030 \text { a favor } \\
\text { de hombre }\end{array}$} \\
\hline & Mujer & 203 & 2.76 & 1.328 & \\
\hline \multirow{2}{*}{ Pulgarcito } & Hombre & 10 & 4.00 & 1.333 & \multirow{2}{*}{$\begin{array}{l}\mathrm{T}=2.693 \mathrm{y} \\
\mathrm{p}=0.008 \text { a favor } \\
\text { de hombre }\end{array}$} \\
\hline & Mujer & 203 & 2.82 & 1.349 & \\
\hline \multirow{2}{*}{ Pinocho } & Hombre & 10 & 3.80 & 1.398 & \multirow{2}{*}{$\begin{array}{l}\mathrm{T}=2.277 \mathrm{y} \\
\mathrm{p}=0.024 \mathrm{a} \text { favor } \\
\text { de hombre }\end{array}$} \\
\hline & Mujer & 203 & 2.78 & 1.378 & \\
\hline \multirow{2}{*}{ Blancanieves } & Hombre & 10 & 3.80 & 1.317 & \multirow{2}{*}{$\begin{array}{l}\mathrm{T}=1.855 \mathrm{y} \\
\mathrm{p}=0.065 \mathrm{no} \\
\text { significativa }\end{array}$} \\
\hline & Mujer & 203 & 2.96 & 1.393 & \\
\hline \multirow{2}{*}{ Bambi } & Hombre & 10 & 3.60 & 1.350 & \multirow{2}{*}{$\begin{array}{l}\mathrm{T}=1.722 \mathrm{y} \\
\mathrm{p}=0.087 \text { no } \\
\text { significativa }\end{array}$} \\
\hline & Mujer & 203 & 2.84 & 1.353 & \\
\hline \multirow{2}{*}{ La lechera } & Hombre & 10 & 3.70 & 1.337 & \multirow{2}{*}{$\begin{array}{l}\mathrm{T}=2.653 \mathrm{y} \\
\mathrm{p}=0.009 \text { a favor } \\
\text { de hombre }\end{array}$} \\
\hline & Mujer & 203 & 2.63 & 1.241 & \\
\hline \multirow[b]{2}{*}{ El mago de Oz } & Hombre & 10 & 4.00 & 1.118 & \multirow{2}{*}{$\begin{array}{l}\mathrm{T}=2.757 \mathrm{y} \\
\mathrm{p}=0.006 \text { a favor } \\
\text { de hombre }\end{array}$} \\
\hline & Mujer & 203 & 2.74 & 1.343 & \\
\hline \multirow{2}{*}{ La bella y la bestia } & Hombre & 10 & 3.70 & 1.337 & \multirow{2}{*}{$\begin{array}{l}\mathrm{T}=1.944 \mathrm{y} \\
\mathrm{p}=0.053 \text { no } \\
\text { significativa }\end{array}$} \\
\hline & Mujer & 203 & 2.85 & 1.348 & \\
\hline \multirow{2}{*}{ El libro de la selva } & Hombre & 10 & 3.80 & 1.317 & \multirow{2}{*}{$\begin{array}{l}\mathrm{T}=1.795 \mathrm{y} \\
\mathrm{p}=0.074 \text { no } \\
\text { significativa }\end{array}$} \\
\hline & Mujer & 203 & 3.30 & 1.252 & \\
\hline
\end{tabular}

Las hombres que participan en este estudio están más de acuerdo que Los tres cerditos $(\mathrm{t}=2.224$ y $\mathrm{p}=0.027,=4.10)$, Hansel $y$ Gretel $(\mathrm{t}=2.339$ y $\mathrm{p}=0.020,=$ 4.00), Pulgarcito $(\mathrm{t}=2.693$ y p $=0.008,=4.00)$, y El Mago de Oz $(\mathrm{t}=2.757$ y p $=$ $0.006,=4.00)$ son los cuentos que mejor transmiten el valor de cooperación y/o ayuda mediante una metodología basada en aspectos tradicionales con respeto a las mujeres.

Mientras que los restantes cuantos infantiles no arrojan diferencias estadísticamente significativas entre la opinión de los participantes en relación a la transmisión del valor cooperación y/o ayuda mediante una metodología basada en aspectos tradicionales y el sexo de los mismos.

Considerando en esta ocasión la edad de la muestra participante, hemos efectuado una Anova (n.s. = 0.05), a la cual se le aplicado previamente el Test de Levene que ha reflejado homogeneidad en las varianzas, obteniendo los siguientes datos distintivos que se exponen en la Tabla 4. 
TABLA 4

Anova en función de la edad de los participantes

\begin{tabular}{|c|c|c|c|c|c|}
\hline Cuento & EDAD & $\mathrm{N}$ & MEDIA & s & F Y P \\
\hline \multirow[t]{6}{*}{ El patito feo } & Menos 25 años & 86 & 3.62 & 1.005 & \multirow{6}{*}{$\begin{array}{c}\mathrm{F}=18.302 \mathrm{y} \mathrm{p}=0.000 \\
\quad \text { favorable a } \\
\text { Menos } 25>31-35 \text { años } \\
\text { Menos } 25>36-40 \text { años } \\
\text { Menos } 25>41-45 \text { años } \\
\text { Menos } 25>\text { Más de } 46\end{array}$} \\
\hline & De 26 a 30 & 10 & 2.70 & 1.160 & \\
\hline & De 31 a 35 & 17 & 2.25 & 1.065 & \\
\hline & De 36 a 40 & 18 & 2.47 & .943 & \\
\hline & De 41 a 45 & 30 & 2.07 & 1.299 & \\
\hline & Más de 46 años & 52 & 2.02 & 1.101 & \\
\hline \multirow[t]{6}{*}{ Caperucita Roja } & Menos 25 años & 86 & 3.55 & 1.011 & \multirow{6}{*}{$\begin{array}{c}\mathrm{F}=15.617 \mathrm{y} \\
\mathrm{p}=0.000 \text { favorable a } \\
\text { Menos } 25>31-35 \text { años } \\
\text { Menos } 25>36-40 \text { años } \\
\text { Menos } 25>41-45 \text { años } \\
\text { Menos } 25>\text { Más de } 46\end{array}$} \\
\hline & De 26 a 30 & 10 & 2.70 & 1.703 & \\
\hline & De 31 a 35 & 17 & 1.94 & 1.181 & \\
\hline & De 36 a 40 & 18 & 2.28 & .752 & \\
\hline & De 41 a 45 & 30 & 2.07 & 1.328 & \\
\hline & Más de 46 años & 52 & 2.17 & 1.098 & \\
\hline \multirow{6}{*}{$\begin{array}{l}\text { El flautista de } \\
\text { Hamelín }\end{array}$} & Menos 25 años & 86 & 3.56 & 1.045 & \multirow{6}{*}{$\begin{array}{c}\mathrm{F}=14.829 \mathrm{y} \\
\mathrm{p}=0.000 \text { favorable a } \\
\text { Menos } 25>31-35 \text { años } \\
\text { Menos } 25>36-40 \text { años } \\
\text { Menos } 25>41-45 \text { años } \\
\text { Menos } 25>\text { Más de } 46\end{array}$} \\
\hline & De 26 a 30 & 10 & 3.10 & 1.370 & \\
\hline & De 31 a 35 & 17 & 2.31 & 1.138 & \\
\hline & De 36 a 40 & 18 & 2.44 & .922 & \\
\hline & De 41 a 45 & 30 & 2.11 & 1.340 & \\
\hline & Más de 46 años & 52 & 2.13 & 1.044 & \\
\hline \multirow[t]{6}{*}{ Hansel y Gretel } & Menos 25 años & 86 & 4.04 & .884 & \multirow{6}{*}{$\begin{array}{c}\mathrm{F}=29.100 \text { y } \mathrm{p}=0.000 \\
\text { favorable a } \\
\text { Menos } 25>31-35 \text { años } \\
\text { Menos } 25>36-40 \text { años } \\
\text { Menos } 25>41-45 \text { años } \\
\text { Menos } 25>\text { Más de } 46 \\
26-30 \text { años }>41-45 \text { años }\end{array}$} \\
\hline & De 26 a 30 & 10 & 3.20 & 1.398 & \\
\hline & De 31 a 35 & 17 & 2.31 & 1.250 & \\
\hline & De 36 a 40 & 18 & 2.56 & 1.097 & \\
\hline & De 41 a 45 & 30 & 1.96 & 1.255 & \\
\hline & Más de 46 años & 52 & 2.09 & 1.158 & \\
\hline \multirow{6}{*}{$\begin{array}{l}\text { La bella } \\
\text { durmiente }\end{array}$} & Menos 25 años & 86 & 3.61 & 1.109 & \multirow{6}{*}{$\begin{array}{c}\mathrm{F}=16.839 \mathrm{y} \\
\mathrm{p}=0.000 \text { favorable a } \\
\text { Menos } 25>31-35 \text { años } \\
\text { Menos } 25>36-40 \text { años } \\
\text { Menos } 25>41-45 \text { años } \\
\text { Menos } 25>\text { Más de } 46\end{array}$} \\
\hline & De 26 a 30 & 10 & 3.00 & 1.333 & \\
\hline & De 31 a 35 & 17 & 2.25 & 1.125 & \\
\hline & De 36 a 40 & 18 & 2.39 & .778 & \\
\hline & De 41 a 45 & 30 & 2.00 & 1.209 & \\
\hline & Más de 46 años & 52 & 2.06 & 1.150 & \\
\hline
\end{tabular}




\begin{tabular}{|c|c|c|c|c|c|}
\hline Cuento & EDAD & $\mathrm{N}$ & MEDIA & s & F Y P \\
\hline \multirow{6}{*}{$\begin{array}{l}\text { El gato con } \\
\text { botas }\end{array}$} & Menos 25 años & 86 & 3.63 & 1.138 & \multirow{6}{*}{$\begin{array}{c}\mathrm{F}=15.878 \text { y } \mathrm{p}=0.000 \\
\quad \text { favorable a } \\
\text { Menos } 25>31-35 \text { años } \\
\text { Menos } 25>36-40 \text { años } \\
\text { Menos } 25>41-45 \text { años } \\
\text { Menos } 25>\text { Más de } 46\end{array}$} \\
\hline & De 26 a 30 & 10 & 2.60 & 1.265 & \\
\hline & De 31 a 35 & 17 & 2.27 & 1.223 & \\
\hline & De 36 a 40 & 18 & 2.67 & 1.138 & \\
\hline & De 41 a 45 & 30 & 2.00 & 1.177 & \\
\hline & Más de 46 años & 52 & 2.06 & 1.131 & \\
\hline \multirow[t]{6}{*}{ Los tres cerditos } & Menos 25 años & 86 & 4.13 & 1.067 & \multirow{6}{*}{$\begin{array}{c}\mathrm{F}=26.493 \text { y } \mathrm{p}=0.000 \\
\text { favorable a } \\
\text { Menos } 25>31-35 \text { años } \\
\text { Menos } 25>36-40 \text { años } \\
\text { Menos } 25>41-45 \text { años } \\
\text { Menos } 25>\text { Más de } 46 \\
\text { 26-30 años }>41-45 \text { años } \\
\text { 26-30 años }>\text { Más de } 46\end{array}$} \\
\hline & De 26 a 30 & 10 & 3.50 & 1.434 & \\
\hline & De 31 a 35 & 17 & 2.56 & 1.413 & \\
\hline & De 36 a 40 & 18 & 2.61 & 1.145 & \\
\hline & De 41 a 45 & 30 & 2.00 & 1.301 & \\
\hline & Más de 46 años & 52 & 2.06 & 1.169 & \\
\hline \multirow[t]{6}{*}{ Peter Pan } & Menos 25 años & 86 & 4.14 & .907 & \multirow{6}{*}{$\begin{array}{c}\mathrm{F}=35.005 \text { y } \mathrm{p}=0.000 \\
\text { favorable a } \\
\text { Menos } 25>31-35 \text { años } \\
\text { Menos } 25>36-40 \text { años } \\
\text { Menos } 25>41-45 \text { años } \\
\text { Menos } 25>\text { Más de } 46 \\
\text { 26-30 años }>41-45 \text { años } \\
\text { 26-30 años }>\text { Más de } 46\end{array}$} \\
\hline & De 26 a 30 & 10 & 3.30 & 1.418 & \\
\hline & De 31 a 35 & 17 & 2.13 & .990 & \\
\hline & De 36 a 40 & 18 & 2.33 & 1.085 & \\
\hline & De 41 a 45 & 30 & 2.11 & 1.340 & \\
\hline & Más de 46 años & 52 & 2.00 & 1.083 & \\
\hline \multirow[t]{6}{*}{ La Cenicienta } & Menos 25 años & 86 & 3.82 & 1.146 & \multirow{6}{*}{$\begin{array}{c}\mathrm{F}=20.616 \text { y } \mathrm{p}=0.000 \\
\text { favorable a } \\
\text { Menos } 25>31-35 \text { años } \\
\text { Menos } 25>36-40 \text { años } \\
\text { Menos } 25>41-45 \text { años } \\
\text { Menos } 25>\text { Más de } 46 \\
\text { 26-30 años }>31-35 \text { años } \\
\text { 26-30 años }>41-45 \text { años } \\
\text { 26-30 años }>\text { Más de } 46\end{array}$} \\
\hline & De 26 a 30 & 10 & 3.60 & 1.506 & \\
\hline & De 31 a 35 & 17 & 2.19 & 1.167 & \\
\hline & De 36 a 40 & 18 & 2.50 & 1.098 & \\
\hline & De 41 a 45 & 30 & 2.22 & 1.340 & \\
\hline & Más de 46 años & 52 & 1.96 & 1.083 & \\
\hline \multirow{6}{*}{$\begin{array}{l}\text { Los siete } \\
\text { cabritillos }\end{array}$} & Menos 25 años & 86 & 3.73 & 1.084 & \multirow{6}{*}{$\begin{array}{c}\mathrm{F}=20.408 \text { y } \mathrm{p}=0.000 \\
\text { favorable a } \\
\text { Menos } 25>31-35 \text { años } \\
\text { Menos } 25>36-40 \text { años } \\
\text { Menos } 25>41-45 \text { años } \\
\text { Menos } 25>\text { Más de } 46 \\
\text { 26-30 años > Más de } 46\end{array}$} \\
\hline & De 26 a 30 & 10 & 3.20 & 1.135 & \\
\hline & De 31 a 35 & 17 & 2.06 & 1.063 & \\
\hline & De 36 a 40 & 18 & 2.50 & 1.098 & \\
\hline & De 41 a 45 & 30 & 2.11 & 1.251 & \\
\hline & Más de 46 años & 52 & 2.02 & 1.093 & \\
\hline
\end{tabular}


EL APRENDIZAJE DE VALORES A TRAVÉS DE LOS CUENTOS EN EDUCACIÓN INFANTIL

\begin{tabular}{|c|c|c|c|c|c|}
\hline Cuento & EDAD & $\mathbf{N}$ & MEDIA & s & F Y P \\
\hline \multirow[t]{6}{*}{ Los tres osos } & Menos 25 años & 86 & 3.61 & 1.120 & \multirow{6}{*}{$\begin{array}{l}\mathrm{F}=14.900 \text { y } \mathrm{p}=0.000 \\
\quad \text { favorable a } \\
\text { Menos } 25>31-35 \text { años } \\
\text { Menos } 25>36-40 \text { años } \\
\text { Menos } 25>41-45 \text { años } \\
\text { Menos } 25>\text { Más de } 46\end{array}$} \\
\hline & De 26 a 30 & 10 & 2.80 & 1.317 & \\
\hline & De 31 a 35 & 17 & 2.06 & .998 & \\
\hline & De 36 a 40 & 18 & 2.61 & 1.243 & \\
\hline & De 41 a 45 & 30 & 2.15 & 1.322 & \\
\hline & Más de 46 años & 52 & 2.09 & 1.100 & \\
\hline \multirow[t]{6}{*}{ Pulgarcito } & Menos 25 años & 86 & 3.75 & 1.063 & \multirow{6}{*}{$\begin{array}{c}\mathrm{F}=18.795 \text { y } \mathrm{p}=0.000 \\
\text { favorable a } \\
\text { Menos } 25>31-35 \text { años } \\
\text { Menos } 25>36-40 \text { años } \\
\text { Menos } 25>41-45 \text { años } \\
\text { Menos } 25>\text { Más de } 46\end{array}$} \\
\hline & De 26 a 30 & 10 & 3.10 & 1.370 & \\
\hline & De 31 a 35 & 17 & 2.33 & 1.345 & \\
\hline & De 36 a 40 & 18 & 2.61 & 1.378 & \\
\hline & De 41 a 45 & 30 & 2.04 & 1.160 & \\
\hline & Más de 46 años & 52 & 2.04 & 1.042 & \\
\hline \multirow[t]{6}{*}{ Pinocho } & Menos 25 años & 86 & 3.62 & 1.225 & \multirow{6}{*}{$\begin{array}{c}\mathrm{F}=13.832 \text { y } \mathrm{p}=0.000 \\
\text { favorable a } \\
\text { Menos } 25>31-35 \text { años } \\
\text { Menos } 25>41-45 \text { años } \\
\text { Menos } 25>\text { Más de } 46\end{array}$} \\
\hline & De 26 a 30 & 10 & 2.70 & 1.418 & \\
\hline & De 31 a 35 & 17 & 2.20 & 1.207 & \\
\hline & De 36 a 40 & 18 & 2.78 & 1.353 & \\
\hline & De 41 a 45 & 30 & 1.96 & 1.126 & \\
\hline & Más de 46 años & 52 & 2.15 & 1.142 & \\
\hline \multirow[t]{6}{*}{ Blancanieves } & Menos 25 años & 86 & 3.84 & 1.072 & \multirow{6}{*}{$\begin{array}{c}\mathrm{F}=16.187 \text { y } \mathrm{p}= \\
0.000 \text { favorable a } \\
\text { Menos } 25>31-35 \text { años } \\
\text { Menos } 25>36-40 \text { años } \\
\text { Menos } 25>41-45 \text { años } \\
\text { Menos } 25>\text { Más de } 46\end{array}$} \\
\hline & De 26 a 30 & 10 & 3.10 & 1.524 & \\
\hline & De 31 a 35 & 17 & 2.50 & 1.155 & \\
\hline & De 36 a 40 & 18 & 2.83 & 1.383 & \\
\hline & De 41 a 45 & 30 & 2.15 & 1.379 & \\
\hline & Más de 46 años & 52 & 2.19 & 1.154 & \\
\hline \multirow[t]{6}{*}{ Bambi } & Menos 25 años & 86 & 3.64 & 1.204 & \multirow{6}{*}{$\begin{array}{c}\mathrm{F}=12.025 \text { y } \mathrm{p}= \\
0.000 \text { favorable a } \\
\text { Menos } 25>31-35 \text { años } \\
\text { Menos } 25>36-40 \text { años } \\
\text { Menos } 25>41-45 \text { años } \\
\text { Menos } 25>\text { Más de } 46\end{array}$} \\
\hline & De 26 a 30 & 10 & 2.80 & 1.476 & \\
\hline & De 31 a 35 & 17 & 2.33 & .976 & \\
\hline & De 36 a 40 & 18 & 2.44 & 1.149 & \\
\hline & De 41 a 45 & 30 & 2.07 & 1.174 & \\
\hline & Más de 46 años & 52 & 2.35 & 1.246 & \\
\hline
\end{tabular}




\begin{tabular}{|c|c|c|c|c|c|}
\hline CUENTO & EDAD & $\mathrm{N}$ & MEDIA & $\mathrm{s}$ & F Y P \\
\hline \multirow[t]{6}{*}{ La lechera } & Menos 25 años & 86 & 3.27 & 1.204 & \multirow{6}{*}{$\begin{array}{c}\mathrm{F}=8.349 \text { y } \mathrm{p}=0.000 \\
\text { favorable a } \\
\text { Menos } 25>41-45 \text { años } \\
\text { Menos } 25>\text { Más de } 46\end{array}$} \\
\hline & De 26 a 30 & 10 & 3.00 & 1.247 & \\
\hline & De 31 a 35 & 17 & 2.40 & .986 & \\
\hline & De 36 a 40 & 18 & 2.44 & 1.149 & \\
\hline & De 41 a 45 & 30 & 2.00 & 1.144 & \\
\hline & Más de 46 años & 52 & 2.17 & 1.129 & \\
\hline \multirow[t]{6}{*}{ El mago de Oz } & Menos 25 años & 86 & 3.48 & 1.266 & \multirow{6}{*}{$\begin{array}{c}\mathrm{F}=9.700 \text { y } \mathrm{p}=0.000 \\
\text { favorable a } \\
\text { Menos } 25>31-35 \text { años } \\
\text { Menos } 25>36-40 \text { años } \\
\text { Menos } 25>41-45 \text { años } \\
\text { Menos } 25>\text { Más de } 46\end{array}$} \\
\hline & De 26 a 30 & 10 & 3.00 & 1.155 & \\
\hline & De 31 a 35 & 17 & 2.44 & 1.315 & \\
\hline & De 36 a 40 & 18 & 2.50 & 1.249 & \\
\hline & De 41 a 45 & 30 & 2.15 & 1.199 & \\
\hline & Más de 46 años & 52 & 2.15 & 1.154 & \\
\hline \multirow{6}{*}{$\begin{array}{l}\text { La bella y } \\
\text { la bestia }\end{array}$} & Menos 25 años & 86 & 3.71 & 1.050 & \multirow{6}{*}{$\begin{array}{c}\mathrm{F}=16.680 \text { y } \mathrm{p}=0.000 \\
\text { favorable a } \\
\text { Menos } 25>31-35 \text { años } \\
\text { Menos } 25>36-40 \text { años } \\
\text { Menos } 25>41-45 \text { años } \\
\text { Menos } 25>\text { Más de } 46\end{array}$} \\
\hline & De 26 a 30 & 10 & 3.30 & 1.418 & \\
\hline & De 31 a 35 & 17 & 2.19 & .911 & \\
\hline & De 36 a 40 & 18 & 2.33 & .840 & \\
\hline & De 41 a 45 & 30 & 2.22 & 1.423 & \\
\hline & Más de 46 años & 52 & 2.15 & 1.268 & \\
\hline \multirow{6}{*}{$\begin{array}{l}\text { El libro de } \\
\text { la selva }\end{array}$} & Menos 25 años & 86 & 4.01 & .988 & \multirow{6}{*}{$\begin{array}{c}\mathrm{F}=23.911 \text { y } \\
\mathrm{p}=0.000 \text { favorable a } \\
\text { Menos } 25>31-35 \text { años } \\
\text { Menos } 25>36-40 \text { años } \\
\text { Menos } 25>41-45 \text { años } \\
\text { Menos } 25>\text { Más de } 46\end{array}$} \\
\hline & De 26 a 30 & 10 & 3.20 & 1.317 & \\
\hline & De 31 a 35 & 17 & 2.19 & 1.109 & \\
\hline & De 36 a 40 & 18 & 2.53 & 1.007 & \\
\hline & De 41 a 45 & 30 & 2.22 & 1.368 & \\
\hline & Más de 46 años & 52 & 2.13 & 1.227 & \\
\hline
\end{tabular}

Los participantes que tienen menos de 25 años señalan estar más de acuerdo con la idea que el cuento El patito feo transmite el valor de cooperación y/o ayuda mediante una metodología con aspectos tradicionales, $\mathrm{F}(5,207)=18.302, \mathrm{p}=0.000$, $\eta 2=0.318$, que los de 31 a 35 años, $\mathrm{t}(207)=4.656, \mathrm{p}=0.000$; los de 36 a 40 años, $\mathrm{t}(207)=4.000, \mathrm{p}=0.001 ;$ los de 41 a 45 años, $\mathrm{t}(207)=6.464, \mathrm{p}=0.000 ; \mathrm{y}$ los de más de 46 años, $\mathrm{t}(207)=8.195, \mathrm{p}=0.000$. Mientras que no existen datos significativos con las restantes comparaciones.

En relación, a la narrativa infantil Caperucita Roja $[\mathrm{F}(5,207)=15.617, \mathrm{p}=$ $0.000, \eta 2=0.284]$, la muestra que tiene menos de 25 años está más de acuerdo con esta idea frente a los de 31 a 35 años, t(207) = 5.296, p = 0.000; los de 36 a 
40 años, $\mathrm{t}(207)=4.394, \mathrm{p}=0.000 ;$ los de 41 a 45 años, $\mathrm{t}(207)=5.992, \mathrm{p}=0.000$; y los de más de 46 años, t $(207)=6.871, \mathrm{p}=0.000$. Sin embargo, las pruebas post hoc aplicadas no referencian datos estadísticamente significativos en el resto de las combinaciones binarias.

El flautista de Hamelín como cuento infantil $[\mathrm{F}(5,207)=14.829, \mathrm{p}=0.000, \eta 2$ $=0.273$ ], según señalan los participantes de menos de 25 años frente a los de 31 a 35 años, t (207) $=4.143, \mathrm{p}=0.001 ;$ los de 36 a 40 años, $\mathrm{t}(207)=3.898, \mathrm{p}=0.002 ;$ los de 41 a 45 años, t(207) = 5.934, p = 0.000; y los de más de 46 años, t $(207)=7.211$, $\mathrm{p}=0.000$; en cambio, no existen datos significativos de Gabriel y Bonferroni con las restantes comparaciones.

Aquellas personas que tienen menos de 25 años están más de acuerdo en que la obra infantil Hansel y Gretel $[\mathrm{F}(5,207)=29.100, \mathrm{p}=0.000, \eta 2=0.426]$; transmite el valor de cooperación y/o ayuda a través de métodos tradicionales, frente a los de 31 a 35 años, $\mathrm{t}(207)=5.840, \mathrm{p}=0.000$; los de 36 a 40 años, $\mathrm{t}(207)=5.266$, $\mathrm{p}$ $=0.000 ;$ los de 41 a 45 años, $\mathrm{t}(207)=8.673, \mathrm{p}=0.000 ; \mathrm{y}$ los de más de 46 años, $\mathrm{t}(207)=9.903, \mathrm{p}=0.000$. De igual forma, los de 26 a 30 años frente a los de $41 \mathrm{a}$ 45 años, $\mathrm{t}(207)=3.093, \mathrm{p}=0.034$; no obstante las pruebas post hoc aplicadas no revelan significatividad en el resto de comparaciones.

Los participantes que tienen menos de 25 años señalan estar más de acuerdo en que la narrativa infantil La bella durmiente transmite el valor de cooperación y/o ayuda a través de métodos tradicionales $[\mathrm{F}(5,207)=16.839, \mathrm{p}=0.000, \eta 2=0.300]$, que de los de 31 a 35 años, t(207) = 4.435, p = 0.000; los de 36 a 40 años, t(207) $=4.186, \mathrm{p}=0.001 ;$ los de 41 a 45 años, $\mathrm{t}(207)=6.479, \mathrm{p}=0.000 ; \mathrm{y}$ los de más de 46 años, $\mathrm{t}(207)=7.564, \mathrm{p}=0.000$. Mientras que no existen datos significativos con las restantes comparaciones.

Al considerar la obra infantil El gato con botas y la transmisión del valor de cooperación y/o ayuda mediante una metodología de aspecto tradicional $[\mathrm{F}(5,207)$ $=15.878, \mathrm{p}=0.000, \eta 2=0.289$ ], la muestra que tiene menos de 25 años está más de acuerdo con esta idea frente a los de los de 31 a 35 años, t(207) $=4.223$, p = 0.001; los de 36 a 40 años, $\mathrm{t}(207)=3.213, \mathrm{p}=0.023$; los de 41 a 45 años, $\mathrm{t}(207)=$ 6.396, $\mathrm{p}=0.000 ; \mathrm{y}$ los de más de 46 años, $\mathrm{t}(207)=7.462, \mathrm{p}=0.000$. Sin embargo, las pruebas post hoc aplicadas no referencia datos estadísticamente significativos en el resto de las combinaciones binarias.

Los tres cerditos como cuento infantil $[\mathrm{F}(5,207)=26.493, \mathrm{p}=0.000, \eta 2=0.402]$, según señalan los participantes de menos de 25 años frente a los de 31 a 35 años, $\mathrm{t}(207)=4.882, \mathrm{p}=0.000 ;$ los de $36 \mathrm{a} 40$ años, $\mathrm{t}(207)=4.977, \mathrm{p}=0.000 ;$ los de 41 a 45 años, $\mathrm{t}(207)=8.188, \mathrm{p}=0.000 ; \mathrm{y}$ los de más de 46 años, $\mathrm{t}(207)=9.654, \mathrm{p}=$ 0.000. Del mismo modo, los participantes de 26 a 30 años frente a los de 41 a 45 años, $\mathrm{t}(207)=3.440, \mathrm{p}=0.011 ; \mathrm{y}$ con los de más de 46 años, $\mathrm{t}(207)=3.502, \mathrm{p}=$ 0.009. En cambio, no existen datos significativos de Gabriel y Bonferroni con las restantes comparaciones.

Aquellas personas que tienen menos de 25 años están más de acuerdo en que la obra infantil Peter Pan $[\mathrm{F}(5,207)=35.005, \mathrm{p}=0.000, \eta 2=0.473]$ transmite 
el valor de cooperación y/o ayuda a través de métodos tradicionales, frente a los de 31 a 35 años, $\mathrm{t}(207)=6.745, \mathrm{p}=0.000$; los de 36 a 40 años, $\mathrm{t}(207)=6.558, \mathrm{p}$ $=0.000 ;$ los de 41 a 45 años, $\mathrm{t}(207)=8.647, \mathrm{p}=0.000 ; \mathrm{y}$ los de más de 46 años, $\mathrm{t}(207)=11.046, \mathrm{p}=0.000$. De igual forma, los de 26 a 30 años frente a los de $41 \mathrm{a}$ 45 años, $\mathrm{t}(207)=3.018, \mathrm{p}=0.043$; y con los de más de 46 años, $\mathrm{t}(207)=3.504, \mathrm{p}$ $=0.008$. No obstante, las pruebas post hoc aplicadas no revelan significatividad en el resto de comparaciones.

La Cenicienta transmite el valor de cooperación y/o ayuda mediante métodos tradicionales $[F(5,207)=20.616, p=0.000, \eta 2=0.343]$, según señalan los participantes que tienen menos de 25 años frente a los de 31 a 35 años, t $(207)=5.112$, p $=0.000 ;$ los de 36 a 40 años, t(207) = 4.341, p = 0.000; los de 41 a 45 años, t(207) = 6.157, $\mathrm{p}=0.000 ; \mathrm{y}$ los de más de 46 años, $\mathrm{t}(207)=8.719, \mathrm{p}=0.000$. De igual forma, los de 26 a 30 años frente a los de 31-35 años, t(207) $=2.981$ y p $=0.049$; de 41 a 45 años, $\mathrm{t}(207)=3.167, \mathrm{p}=0.027$; y con los de más de 46 años, $\mathrm{t}(207)=4.017$, $\mathrm{p}$ $=0.001$. En cambio, no existen datos significativos de Gabriel y Bonferroni con las restantes comparaciones.

Los participantes que tienen menos de 25 años indican estar más de acuerdo en que la narrativa infantil Los siete cabritillos transmite el valor de cooperación y/o ayuda mediante métodos tradicionales, $\mathrm{F}(5,207)=20.408, \mathrm{p}=0.000, \eta 2=0.341$, que los de 31 a 35 años, $\mathrm{t}(207)=5.502, \mathrm{p}=0.000$; los de 36 a 40 años, $\mathrm{t}(207)=$ 4.267, $\mathrm{p}=0.000$; los de 41 a 45 años, $\mathrm{t}(207)=6.577, \mathrm{p}=0.000 ;$ y los de más de 46 años, $\mathrm{t}(207)=8.455, \mathrm{p}=0.000$. Finalmente, la comparativa entre aquellas personas de 26 a 30 años frente a los de más de 46 años, t(207) $=3.046, p=0.040$, donde los de menor edad están más de acuerdo con esta idea. Mientras que no existen datos relevantes con las restantes comparaciones.

Al considerar la obra infantil Los tres osos y la transmisión del valor de cooperación y/o ayuda mediante una metodología tradicional $[\mathrm{F}(5,207)=14.900, \mathrm{p}=$ 0.000, $\eta 2=0.275$, los sujetos que tienen menos de 25 años están más de acuerdo con esta premisa que los de 31 a 35 años, $\mathrm{t}(207)=4.905, \mathrm{p}=0.000$; los de $36 \mathrm{a}$ 40 años, $\mathrm{t}(207)=3.220, \mathrm{p}=0.000$; los de 41 a 45 años, $\mathrm{t}(207)=5.699, \mathrm{p}=0.000$; y los de más de 46 años, t $(207)=7.213, \mathrm{p}=0.000$. Sin embargo, las pruebas post hoc aplicadas no referencian datos estadísticamente significativos en el resto de las combinaciones binarias.

Los participantes que tienen menos de 25 años señalan estar más de acuerdo con la idea de que el cuento Pulgarcito transmite el valor de cooperación y/o ayuda mediante una metodología con aspectos tradicionales $[\mathrm{F}(5,207)=18.795, \mathrm{p}$ $=0.000, \eta 2=0.325]$, que los de 31 a 35 años, $\mathrm{t}(207)=4.442, \mathrm{p}=0.000$; los de 36 a 40 años, t (207) $=3.848, \mathrm{p}=0.002$; los de 41 a 45 años, $\mathrm{t}(207)=6.798, \mathrm{p}=0.000$; y los de más de 46 años, $\mathrm{t}(207)=8.206, \mathrm{p}=0.000$. Mientras que no existe datos relevantes con las restantes comparaciones.

Las personas que tienen menos de 25 años frente a los que tienen de 31 a 35 años, $\mathrm{t}(207)=4.188, \mathrm{p}=0.001 ;$ los de 41 a 45 años, $\mathrm{t}(207)=6.197, \mathrm{p}=0.000 ; \mathrm{y}$ los de más de 46 años, $\mathrm{t}(207)=6.674, \mathrm{p}=0.000$ consideran estar más de acuerdo 
con la transmisión del valor cooperación y/o ayuda en la narrativa de Pinocho $[\mathrm{F}(5,207)=13.832, \mathrm{p}=0.000, \eta 2=0.261]$, a través de una metodología tradicional. No obstante, ninguna de las pruebas post hoc aplicadas reflejan significatividad en las restantes comparaciones.

En relación a la narrativa infantil Blancanieves $[\mathrm{F}(5,207)=16.187, \mathrm{p}=0.000, \eta 2$ $=0.290$ ], la muestra que tiene menos de 25 años está más de acuerdo con esta idea frente a los de 31 a 35 años, $\mathrm{t}(207)=4.114, \mathrm{p}=0.001$; los de 36 a 40 años, $\mathrm{t}(207)$ $=3.249, \mathrm{p}=0.021 ;$ los de 41 a 45 años, $\mathrm{t}(207)=6.422, \mathrm{p}=0.000 ; \mathrm{y}$ los de más de 46 años, $\mathrm{t}(207)=7.620, \mathrm{p}=0.000$. Sin embargo, las pruebas post hoc aplicadas no referencian datos estadísticamente significativos en el resto de las combinaciones binarias.

Aquellas personas que tienen menos de 25 años están más de acuerdo en que la obra infantil Bambi transmite el valor de cooperación y/o ayuda mediante metodologías tradicionales $[\mathrm{F}(5,207)=12.025, \mathrm{p}=0.000, \eta 2=0.234]$, que los de 31 a 35 años, $\mathrm{t}(207)=3.863, \mathrm{p}=0.002$; los de 36 a 40 años, $\mathrm{t}(207)=3.805, \mathrm{p}=0.003$; los de 41 a 45 años, t(207) = 5.868, p = 0.000; y los de más de 46 años, t $(207)=5.876$, $\mathrm{p}=0.000$; no obstante las pruebas post hoc aplicadas no revelan significatividad en las restantes comparaciones.

Los participantes que tienen menos de 25 años indican estar más de acuerdo con la idea de que el cuento La lechera transmite el valor de cooperación y/o ayuda mediante una metodología con aspectos tradicionales, $\mathrm{F}(5,207)=8.349, \mathrm{p}=0.000$, $\eta 2=0.178$, que los de 41 a 45 años, $\mathrm{t}(207)=4.930, \mathrm{p}=0.000 ; \mathrm{y}$ los de más de 46 años, $\mathrm{t}(207)=5.169, \mathrm{p}=0.000$. Sin embargo, ninguna de las pruebas post hoc aplicadas reflejan relevancia en las restantes comparaciones.

El mago de $\mathrm{Oz}$, como cuento infantil, transmite el valor de cooperación y/o ayuda a través de metodologías tradicionales, $\mathrm{F}(5,207)=9.700, \mathrm{p}=0.000, \eta 2=$ 0.199, según señalan los sujetos que tienen menos de 25 años frente a los de 31 a 35 años, t (207) = 3.101, $\mathrm{p}=0.034$; los de 36 a 40 años, $\mathrm{t}(207)=3.059, \mathrm{p}=0.038$; los de 41 a 45 años, t(207) = 4.882, $\mathrm{p}=0.000 ; \mathrm{y}$ los de más de 46 años, $\mathrm{t}(207)=5.858$, $\mathrm{p}=0.000$; en cambio, no existen datos significativos de Gabriel y Bonferroni con las restantes combinaciones.

Al considerar la obra infantil La bella y la Bestia y la transmisión del valor de cooperación y/o ayuda mediante una metodología con aspectos tradicionales, $\mathrm{F}(5,207)=16.680, \mathrm{p}=0.000, \eta 2=0.296$, los participantes que tienen menos de 25 años están más de acuerdo con esta idea frente a los de 31 a 35 años, t(207) = 4.847, $\mathrm{p}=0.000$; los de 36 a 40 años, $\mathrm{t}(207)=4.602, \mathrm{p}=0.000$; los de 41 a 45 años, $\mathrm{t}(207)=5.854, \mathrm{p}=0.000 ; \mathrm{y}$ los de más de 46 años, $\mathrm{t}(207)=7.464, \mathrm{p}=0.000$. Sin embargo, las pruebas post hoc aplicadas no referencian datos significativos en el resto de las combinaciones binarias.

Los participantes que tienen menos de 25 años señalan estar más de acuerdo en que la narrativa infantil El libro de la selva transmite el valor de cooperación y/o ayuda a través de métodos tradicionales, $\mathrm{F}(5,207)=23.911, \mathrm{p}=0.000, \eta 2=0.380$, que los de 31 a 35 años, t(207) = 5.922, p = 0.000; los de 36 a 40 años, t(207) = 4.923, 
$\mathrm{p}=$ 0.000; los de 41 a 45 años, t(207) = 7.160, p = 0.000; y los de más de 46 años, $\mathrm{t}(207)=9.145, \mathrm{p}=0.000$. Mientras que no existen datos significativos con las restantes comparaciones.

Al ejecutar la prueba inferencial T de Student (n.s. = 0.05), entre la opinión de los participantes en relación a la transmisión del valor cooperación y/o ayuda en los cuentos mediante una metodología con aspectos tradicionales y la situación profesional de los mismos (ver tabla 5).

\section{TABLA 5}

T de Student en función de la situación profesional de los participantes

\begin{tabular}{|c|c|c|c|c|c|}
\hline CUENTO & S. PROFESIONAL & $\mathrm{N}$ & MEDIA & $\mathrm{s}$ & T Y P \\
\hline \multirow{2}{*}{ El patito feo } & Estudiante & 91 & 3,63 & 1,004 & \multirow{2}{*}{$\begin{array}{l}\mathrm{T}=10.206 \text { y } \mathrm{p}=0.000 \mathrm{a} \text { favor } \\
\text { de estudiante }\end{array}$} \\
\hline & Profesor & 121 & 2,11 & 1,085 & \\
\hline \multirow{2}{*}{$\begin{array}{l}\text { Caperucita } \\
\text { Roja }\end{array}$} & Estudiante & 91 & 3,54 & 1,056 & \multirow{2}{*}{$\begin{array}{l}\mathrm{T}=9.293 \text { y } \mathrm{p}=0.000 \text { a favor } \\
\text { de estudiante }\end{array}$} \\
\hline & Profesor & 121 & 2,11 & 1,113 & \\
\hline \multirow{2}{*}{$\begin{array}{l}\text { El flautista } \\
\text { de Hamelín }\end{array}$} & Estudiante & 91 & 3,54 & 1,067 & \multirow{2}{*}{$\begin{array}{l}\mathrm{T}=8.462 \text { y } \mathrm{p}=0.000 \mathrm{a} \text { favor } \\
\text { de estudiante }\end{array}$} \\
\hline & Profesor & 121 & 2,22 & 1,124 & \\
\hline \multirow{2}{*}{$\begin{array}{l}\text { Hansel y } \\
\text { Gretel }\end{array}$} & Estudiante & 91 & 4,01 & 0,923 & \multirow{2}{*}{$\begin{array}{l}\mathrm{T}=12.312 \text { y } \mathrm{p}=0.000 \mathrm{a} \text { favor } \\
\text { de estudiante }\end{array}$} \\
\hline & Profesor & 121 & 2,18 & 1,187 & \\
\hline \multirow{2}{*}{$\begin{array}{l}\text { La bella } \\
\text { durmiente }\end{array}$} & Estudiante & 91 & 3,62 & 1,103 & \multirow{2}{*}{$\begin{array}{l}\mathrm{T}=9.661 \text { y } \mathrm{p}=0.000 \mathrm{a} \text { favor } \\
\text { de estudiante }\end{array}$} \\
\hline & Profesor & 121 & 2,12 & 1,088 & \\
\hline \multirow{2}{*}{$\begin{array}{l}\text { El gato } \\
\text { con botas }\end{array}$} & Estudiante & 91 & 3,61 & 1,129 & \multirow{2}{*}{$\begin{array}{l}\mathrm{T}=8.901 \text { y } \mathrm{p}=0.000 \text { a favor } \\
\text { de estudiante }\end{array}$} \\
\hline & Profesor & 121 & 2,16 & 1,159 & \\
\hline \multirow{2}{*}{$\begin{array}{l}\text { Los tres } \\
\text { cerditos }\end{array}$} & Estudiante & 91 & 4,14 & 1,076 & \multirow{2}{*}{$\begin{array}{l}\mathrm{T}=11.735 \text { y } \mathrm{p}=0.000 \mathrm{a} \text { favor } \\
\text { de estudiante }\end{array}$} \\
\hline & Profesor & 121 & 2,21 & 1,226 & \\
\hline \multirow{2}{*}{ Peter Pan } & Estudiante & 91 & 4,16 & 0,896 & \multirow{2}{*}{$\begin{array}{l}\mathrm{T}=13.971 \text { y } \mathrm{p}=0.000 \text { a favor } \\
\text { de estudiante }\end{array}$} \\
\hline & Profesor & 121 & 2,11 & 1,126 & \\
\hline \multirow{2}{*}{$\begin{array}{l}\text { La } \\
\text { Cenicienta }\end{array}$} & Estudiante & 91 & 3,84 & 1,151 & \multirow{2}{*}{$\begin{array}{l}\mathrm{T}=10.115 \text { y } \mathrm{p}=0.000 \mathrm{a} \text { favor } \\
\text { de estudiante }\end{array}$} \\
\hline & Profesor & 121 & 2,17 & 1,185 & \\
\hline \multirow{2}{*}{$\begin{array}{l}\text { Los siete } \\
\text { cabritillos }\end{array}$} & Estudiante & 91 & 3,71 & 1,084 & \multirow{2}{*}{$\begin{array}{l}\mathrm{T}=9.914 \text { y } \mathrm{p}=0.000 \mathrm{a} \text { favor } \\
\text { de estudiante }\end{array}$} \\
\hline & Profesor & 121 & 2,15 & 1,133 & \\
\hline \multirow{2}{*}{ Los tres osos } & Estudiante & 91 & 3,57 & 1,137 & \multirow{2}{*}{$\begin{array}{l}\mathrm{T}=8.362 \text { y } \mathrm{p}=0.000 \text { a favor } \\
\text { de estudiante }\end{array}$} \\
\hline & Profesor & 121 & 2,20 & 1,177 & \\
\hline \multirow{2}{*}{ Pulgarcito } & Estudiante & 91 & 3,74 & 1,067 & \multirow{2}{*}{$\begin{array}{l}\mathrm{T}=9.509 \text { y } \mathrm{p}=0.000 \text { a favor } \\
\text { de estudiante }\end{array}$} \\
\hline & Profesor & 121 & 2,20 & 1,192 & \\
\hline
\end{tabular}


EL APRENDIZAJE DE VALORES A TRAVÉS DE LOS CUENTOS EN EDUCACIÓN INFANTIL

\begin{tabular}{|c|c|c|c|c|c|}
\hline CUENTO & S. PROFESIONAL & $\mathrm{N}$ & MEDIA & $\mathrm{s}$ & T Y P \\
\hline \multirow{2}{*}{ Pinocho } & Estudiante & 91 & 3,62 & 1,220 & \multirow{2}{*}{$\begin{array}{l}\mathrm{T}=8.311 \text { y } \mathrm{p}=0.000 \text { a favor } \\
\text { de estudiante }\end{array}$} \\
\hline & Profesor & 121 & 2,20 & 1,192 & \\
\hline \multirow{2}{*}{ Blancanieves } & Estudiante & 91 & 3,85 & 1,074 & \multirow{2}{*}{$\begin{array}{l}\mathrm{T}=9.242 \text { y } \mathrm{p}=0.000 \text { a favor } \\
\text { de estudiante }\end{array}$} \\
\hline & Profesor & 121 & 2,31 & 1,252 & \\
\hline \multirow{2}{*}{ Bambi } & Estudiante & 91 & 3,66 & 1,187 & \multirow{2}{*}{$\begin{array}{l}\mathrm{T}=8.486 \text { y } \mathrm{p}=0.000 \mathrm{a} \text { favor } \\
\text { de estudiante }\end{array}$} \\
\hline & Profesor & 121 & 2,26 & 1,156 & \\
\hline \multirow{2}{*}{ La lechera } & Estudiante & 91 & 3,28 & 1,191 & \multirow{2}{*}{$\begin{array}{l}\mathrm{T}=6.454 \text { y } \mathrm{p}=0.000 \mathrm{a} \text { favor } \\
\text { de estudiante }\end{array}$} \\
\hline & Profesor & 121 & 2,21 & 1,118 & \\
\hline \multirow{2}{*}{$\begin{array}{l}\text { El mago } \\
\text { de Oz }\end{array}$} & Estudiante & 91 & 3,45 & 1,243 & \multirow{2}{*}{$\begin{array}{l}\mathrm{T}=6.756 \text { y } \mathrm{p}=0.000 \mathrm{a} \text { favor } \\
\text { de estudiante }\end{array}$} \\
\hline & Profesor & 121 & 2,27 & 1,213 & \\
\hline \multirow{2}{*}{$\begin{array}{l}\text { La bella y } \\
\text { la bestia }\end{array}$} & Estudiante & 91 & 3,71 & 1,057 & \multirow{2}{*}{$\begin{array}{l}\mathrm{T}=9.355 \text { y } \mathrm{p}=0.000 \mathrm{a} \text { favor } \\
\text { de estudiante }\end{array}$} \\
\hline & Profesor & 121 & 2,21 & 1,196 & \\
\hline \multirow{2}{*}{$\begin{array}{l}\text { El libro de } \\
\text { la selva }\end{array}$} & Estudiante & 91 & 4,00 & 1,000 & \multirow{2}{*}{$\begin{array}{l}\mathrm{T}=11.205 \mathrm{y} \mathrm{p}=0.000 \mathrm{a} \text { favor } \\
\text { de estudiante }\end{array}$} \\
\hline & Profesor & 121 & 2,23 & 1,196 & \\
\hline
\end{tabular}

En lo que se refiere a la situación profesional de los encuestados, podemos decir que la misma es una variable que refleja resultados estadísticamente significativos en todos los cuentos, siendo la opinión de los estudiantes de Ciencias de la Educación más favorable a la idea de que las 19 obras infantiles transmiten el valor de cooperación y/o ayuda mediante métodos tradicionales.

Así mismo, grosso modo, podemos señalar que los estudiantes participantes en esta investigación son los que están más de acuerdo con que Peter Pan $(\mathrm{t}=13.971$ y $\mathrm{p}=0.000,=4.16)$, Los tres cerditos $(\mathrm{t}=11.735$ y $\mathrm{p}=0.000,=4.14)$, Hansel y Gretel $(\mathrm{t}=12.312$ y $\mathrm{p}=0.000,=4.01)$, y El libro de la selva $(\mathrm{t}=11.205 \mathrm{y}$ $\mathrm{p}=0.000,=4.00)$ son cuentos que transmiten el valor de cooperación y/o ayuda mediante una metodología basada en aspectos tradicionales con respecto a los profesores en ejercicio encuestados.

De igual modo, aunque con menos puntuación, los estudiantes, encuestados, del Grado de Educación Infantil de la Universidad de Córdoba, están de acuerdo con que las siguientes obras infantiles: Blancanieves $(\mathrm{t}=9.242$ y p $=0.000,=3.85)$, La Cenicienta $(\mathrm{t}=10.115$ y $\mathrm{p}=0.000,=3.84)$, Pulgarcito $(\mathrm{t}=9.509$ y $\mathrm{p}=0.000$, $=3.74)$, Los siete cabritillos $(\mathrm{t}=9.914$ y $\mathrm{p}=0.000,=3.71)$, La bella y la bestia $(\mathrm{t}=9.355$ y $\mathrm{p}=0.000,=3.71)$, Bambi $(\mathrm{t}=8.486$ y p $=0.000,=3.66)$, El patito feo $(\mathrm{t}=10.206$ y $\mathrm{p}=0.000,=3.63)$, La bella durmiente $(\mathrm{t}=9.661 \mathrm{y} \mathrm{p}=0.000,=3.62)$, Pinocho $(\mathrm{t}=8.311$ y p $=0.000,=3.62)$, El gato con botas $(\mathrm{t}=8.901$ y $\mathrm{p}=0.000$, $=3.61)$, Los tres osos $(\mathrm{t}=8.362$ y $\mathrm{p}=0.000,=3.57)$, Caperucita Roja $(\mathrm{t}=9.293 \mathrm{y}$ $\mathrm{p}=0.000,=3.54)$, y El flautista de Hamelin $(\mathrm{t}=8.462$ y $\mathrm{p}=0.000,=3.54)$ transmiten 
el valor de cooperación y/o ayuda a través de métodos tradicionales, en comparación con los docentes en ejercicio.

Por otro lado, señalan, estos estudiantes, de manera indiferente, los cuentos infantiles El mago de $\mathrm{Oz}(\mathrm{t}=6.756$ y $\mathrm{p}=0.000,=3.45)$, y La lechera $(\mathrm{t}=6.454 \mathrm{y}$ $\mathrm{p}=0.000,=3.28)$, como recursos que transmiten el valor de cooperación y/o ayuda mediante métodos tradicionales frente a los profesores que están en activo en su profesión, que indican no estar de acuerdo con esta idea.

\section{CONCLUSiOnes}

El aprendizaje de un valor concreto, como ya se ha indicado anteriormente, puede venir determinado por diferentes factores, que en buena lid se conjugan para que este sea adquirido por el sujeto. En lo que se refiere a la etapa de Educación Infantil, hoy en día la combinación de metodologías tradicionales con otras, como son las apoyadas en recursos digitales, puede supeditar dicha transmisión.

Atendiendo al interrogante de partida (¿cuál es la opinión de los docentes, en ejercicio y en formación inicial final, de la etapa de Educación Infantil sobre el valor de los cuentos a través de metodologías tradicionales en la transmisión de valores de los menores?), podemos considerar que los cuentos tradicionales ayudan a que el valor de cooperación y/o ayuda mutua pueda ser adquirido por los niños que cursan la etapa de Educación Infantil. Hay que destacar que tanto hombres como mujeres consideran, en general, que todos los cuentos seleccionados potencian el aprendizaje de este valor a través de metodologías tradicionales, destacando los cuentos de Los tres cerditos, Hansel y Gretel, Pulgarcito y El mago de Oz.

Con respecto al objetivo general y al específico para el valor estudiado, podemos señalar que los maestros en preservicio son más abiertos a la hora de indicar sus opiniones, decantándose por ver que todos los cuentos pueden desarrollar el aprendizaje del valor de la cooperación y/o ayuda mutua. Es significativo que los docentes de más edad vayan indicando valores menores en la consecución del aprendizaje de la colaboración y/o ayuda mutua según se avanza en esta.

Tratando de dilucidar si no es solo la edad la que pude determinar dichas opiniones, sino que puede estar mediatizada por los años de experiencia profesional, nos encontramos la misma situación, la nula experiencia indica una apertura de expectativas y la máxima indica que el objeto se ha difuminado a través del tiempo, con lo cual se abre una doble vía de investigación que determine, por un lado, si la utilización de otras metodologías implicaría una mayor apertura y si esta vendría supeditada por los años de experiencia profesional o la edad.

En consecuencia, podemos concluir que los futuros docentes o maestros en preservicio se encuentran más abiertos al empleo de los cuentos en las aulas, utilizando las metodologías tradicionales de cara al aprendizaje de los valores socialmente aceptados. 


\section{REFERENCIAS BIBLIOGRÁFICAS}

Duque, C. y Ovalle, A. (2012). El docente como mediador entre el niño y el texto narrativo para la comprensión de valores en contextos de interacción en el aula. Revista Perspectivas Educativas, 5, 63-82. Recuperado de http://revistas.ut.edu.co/index.php/ perspectivasedu/article/viewFile/684/529.

Gutiérrez, J. A. (2016). El cuento como herramienta de motivación a la lectoescritura en Educación Primaria: la realidad cercana como inspiración. PublicacionesDidácticas. com, 70, 98-100.

Lawence, R. L. y Pauge, D. S. (2016). What Our Ancestors Knew: Teaching and Learning Through Storytelling. New Directions for Adult and Continuing Education, 149, 63-67. doi: 10.1002/ace.20177.

Mateo, J. (2012). La investigación ex post-facto. En R. Bisquerra (Coord.). Metodología de la investigación educativa (pp. 195-229). Madrid: La Muralla.

Morales, P. (2007). La fiabilidad de los test y las escalas. Madrid: Universidad Pontificia Comillas. Recuperado de http://web.upcomillas.es/personal/peter/estadisticabasica/ Fiabilidad.pdf.

Padial, R. y Saénz-López, P. (2013). Los cuentos populares/tradicionales en educación infantil. Una propuesta a través del juego. E-motion, Revista de Educación, Motricidad e Investigación, 2, 37-47.

Parra, J. M. (2003). La educación en valores y su práctica en el aula. Tendencias Pedagógicas, 8, 69-88.

Sabariego, M. (2012). La investigación educativa: génesis, evolución y características. En R. Bisquerra (Coord.). Metodología de la investigación educativa (pp. 51-87). Madrid: La Muralla.

Sáez, F. (2015). Educación en valores y comunicación de masas: un replanteamiento del problema. ZER, 20 (38), 129-141. 